\title{
Video Transmission over Mobile Adhoc Network Using WEAC Protocol with Zone Routing Algorithm
}

\author{
A. Kamatchi, Dr. Annasaro Vijendran \\ Ph.D( Part time) Scholar, Department of Computer Science, Karpagam University, Coimbatore. \\ Director, MCA Department, SNR \& Sons College, Coimbatore.
}

\begin{abstract}
In mobile adhoc network (MANET), a node communicates directly with the nodes within wireless range and indirectly with other nodes using a dynamically computed, multi-hop route via the other nodes of the manet. In order to facilitate communication within the network, a routing protocol is used to discover routes between nodes. The primary goal of such an adhoc network routing protocol is correct and efficient route establishment between a pair of nodes so that messages may be delivered in a timely manner. Zone Routing based on Zone Routing Protocol (ZRP) is the famous techniques which is used to provide better result in Video transmission. Video transmission in this area is became popular and useful technique. This paper introduces WEAC-VBS-O Protocol with Zone Routing Algorithm. In this work WEAC Protocol with Zone Routing algorithm is discussed. It provides better result for video transmission.
\end{abstract}

Keywords: MANET, Video Transmission, Zone Routing algorithm, WEAC Protocol.

\section{Introduction}

Recently, wireless technology has been one of the hottest topics in computing and communications. Since the late 1970s, consumer wireless applications such as mobile phones began to take off, and presently people are beginning to activate third generation (3G) networks for commercial purposes. Wireless networking technology offering high data rates for mobile users will flourish which will enable the handling of multimedia Web content, videoconferencing, and e-commerce, etc. Routing is one of the key issues for supporting these demanding applications in a rather unstable and resource limited wireless networking environment. There are two ways to implement mobile wireless networks - infrastructured network and infrastructureless (adhoc) network[1]. With an infrastructured network, mobile nodes communicate only with the base stations providing interrnode routing and fixed network connectivity[2]. With the infrastructureless mobile network, each node communicates with other nodes directly or indirectly through intermediate nodes. Thus, all nodes are virtually routers participating in some protocol required for deciding and maintaining the routes[3].

A large number of routing protocols have been developed for mobile adhoc networks (MANETs) ], which is characterized by unpredictable network topology changes, high degree of mobility, energy constrained mobile nodes, bandwidth constrained, intermittent connection, and Memory constrained. The routing problem has been well researched in infrastructure wireless networks, where the goals are efficient detection and adaptation to the network topology, scalability, and convergence. Even though these are equally valid for MANETs, the solutions are more difficult to find since MANETs are inherently more dynamic[4][5].

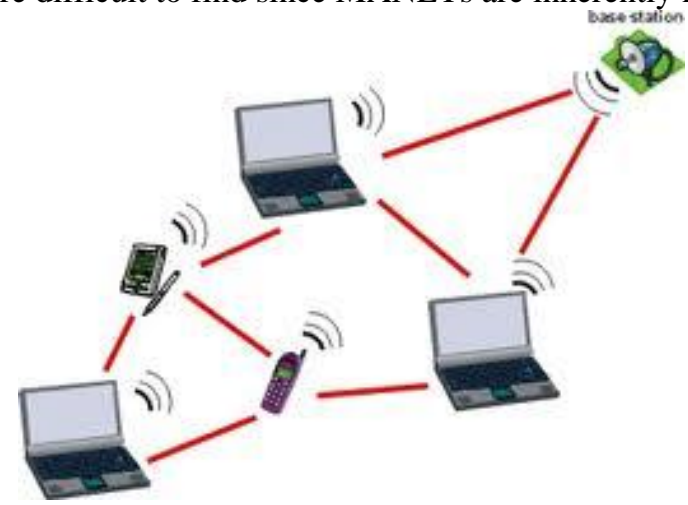

Fig. 1: A Mobile Adhoc Network

In recent years MANET has gained popularity and lots of research is being done on different aspects of MANET. It is an infrastructure less network having no fixed base stations MANET is characterized by dynamic topology low bandwidth and low power consumption. All the nodes in the network are moving i.e. topology of the network is dynamic so the nodes can act both as host as well as router to route information unnecessary for its use. This kind of infrastructure-less network is very useful in situation in which ordinary wired networks is 
not feasible like battlefields, natural disasters etc. The nodes which are in the transmission range of each other communicate directly otherwise communication is done through intermediate nodes which are willing to forward packet hence these networks are also called as multi-hop networks[6][7].

\section{Routing Protocols For Ad Hoc Networks}

The routing protocols proposed for MANETs are generally categorized as Table- driven, sourceinitiated on-demand driven, and hybrid based on the timing when the routes are updated. With the table -driven routing protocols, each node attempts to maintain consistent, up -to-date routing information to every other node in the network. With source initiated on - demand routing, route discovery and maintenance are performed only when a source node desires them[8]. The hybrid approach combines the two approaches to minimize the overhead incurred during route discovery and maintenance. In this section, the protocols belonging to each of the three aforementioned categories are discussed.

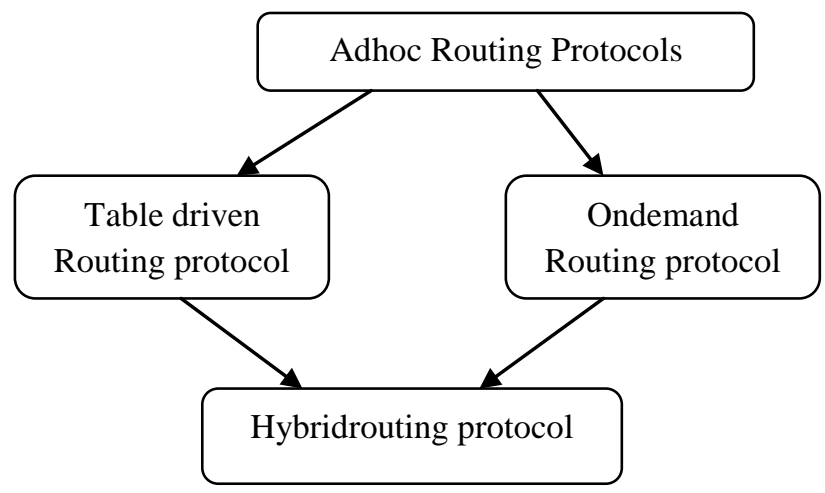

Fig.2 : Types of Routing Protocol

\subsection{Table Driven Routing Protocols}

In table-driven routing protocols, each node maintains uptodate routing table by responding to the changes in network topology and propagating the updates. Thus, it is proactive in the sense that when a packet needs to be forwarded the route is already known and can be immediately used[9][10]. As is the case for wired networks, each node in a MANET maintains a routing table containing a list of all the destinations, next hop, and the number of hops to each destination. The routing table is constructed using either link-state or distance vector algorithms. There are a number of protocols that belong to this category, which are different in the number of tables manipulated for routing and the methods used for exchanging and maintaining routing tables.

Example: DSDV, OLSR, WRP

\subsection{Source-Initiated On -Demand Driven Protocols}

These are reactive protocols where routes are created only when desired by the source node[11]. The two basic procedures of source -initiated on-demand driven protocols are route discovery process and route maintenance process. The route discovery process involves sending route -request packets to neighbor nodes, which then forward the request to their neighbors, and so on. Once the route -request reaches the destination or the intermediate node with a "fresh enough" route, the destination/intermediate node responds by unicasting a route -Reply packet back to the neighbor from which it first received the route -request. Once the route is established, it is maintained by some form of route maintenance process until either the destination becomes inaccessible along any path from the source or the route is no longer desired. In contrast to table-driven routing protocols, not all uptodate routes are maintained at every node.

\section{WEAC- VBS-ONDEMAND Protocol}

The Warning Energy Aware Clusterhead (WEAC)/Virtual Base Station On-demand (VBS-O) routing protocol is proposed in this paper. VBS-O runs on top of the WEAC protocol and it acts similar to a local base station in WLANs.[12] In brief, a mobile node is elected from a set of nominees to act as a temporary base station for a period of time within its zone. In each cluster, a token is used to assign the channel among contending Mobile Terminals (MTs).

A clusterhead supports multiple classes of services and also manages to minimize collisions. Here the characteristics and performance of the WEAC protocol are studied and evaluated by simulation. It is shown that both it scales well to large networks of mobile stations and is proved to perform well for non-real time traffic. In this paper, we extend the work presented in to optimize the protocol for video data. Along with routing 
management, we also introduce power and mobility management to further enhance MANET performance for video data[13][14].

\section{VBS Concept}

The concept of a Virtual Base Station VBS electionas shown by [15] is to ensure that the routing and theResource Reservation are achieved and managedthrough the connection. Therefore, this related studyhas invented a VBS node to be a central coordinatingpoint. A VBS must be intelligent enough to act asboth:

1. A host mobile device (transmitting source or destination device) and

2. A routing device (to route or relay received packets from other nodes until reaching their final destination node in MANET).

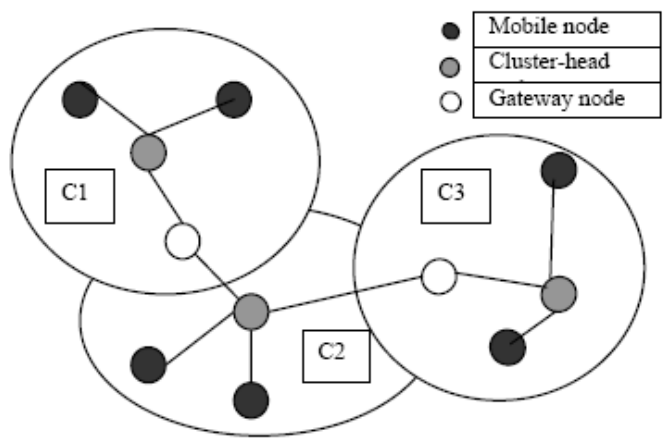

Fig. 3.A typical Cluster-head Network.

According to figure (3) showing a MANET that is divided into three clusters. Each cluster has a "cluster-head" node. The Mobile nodes communicate

through their cluster-head node within the same cluster. Each cluster has a "Gateway" node that connects the cluster "virtually" to the other clustersgateways to enable nodes from different clusters tocommunicate and exchange packets[10].

The previous work on VBS election has suggested a Power-Aware Virtual Base Station (PA-VBS) election algorithm to choose the convenient node to act as a cluster-head (VBS) at a given time for each cluster of nodes based on its battery residual to reduce energy consumption[16][17].

\section{WEAC Protocol With Zone Routing}

As its name implies, the Zone Routing is based on theconcept of zones. Each node is defined with a separate routing zone and zones of neighboring nodes overlap. The routing zone has a radius, $\boldsymbol{r}$, expressed in hops. The zone thus includes those nodes whose distance is at most $\boldsymbol{r}$ hops from the center node. It should however be noted that the zone is defined in hops, not as physical distance. The nodes of a zone are divided into peripheral nodes and interior nodes.[18]

- Peripheral nodesare nodes whose minimum distance to the central node is exactly equal to the zone radius $\boldsymbol{r}$.

- Interior nodesare nodes whose minimum distance is less than $\boldsymbol{r}$.

The construction of a routing zone requires a node to first know who its neighbors are. A neighbor is defined as a node that can communicate directly with the node which is one hop away. Identification of a node's neighbors may be provided directly by the Media Access Control (MAC) protocols[19].

The Zone Routing refers to the locally proactive routing component as the IntrA-zone Routing (IAR). The globally reactive routing component is named IntEr-zone Routing (IER). IER and IAR are not specific routing Methodologies. Instead, IAR is a family of limited-depth, proactive link-state routing protocols. IAR maintains routing information for nodes that are within the routing zone of the node. Correspondingly, IER is a family of reactive routing that offer enhanced route discovery and route maintenance services based on local connectivity monitored by IAR. The fact that the topology of the local zone of each node is known can be used to reduce traffic when global route discovery is needed[20].

ZRP limits the routing information exchange in routing zone, and reduces global route query flooding bybordercasting the query directly to the farthest nodes from the source in a routing zone. This results in the query flowing outward while reducing routing control message overhead. Because of the properties of the proactive routing, node movements cause nearly no extra overhead to maintain. The information of a broken link is only propagated within the zone-radius. 
Zone Routing approach is used in WEAC -VBS-O Protocol to increase the performance while transmitting video packets. It gives improved results because the responsibility for transferring packet from source to destination is handover to the zone leader. So that time taken for transmission also reduced. In this paper this Zone Routing algorithm is used along with WEAC-VBS ) protocol.

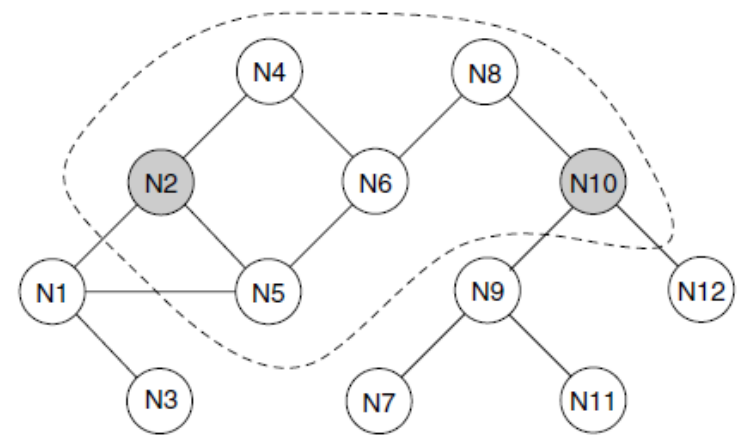

Fig.4. Routing zone and peripheral nodes with routing radius is 2.

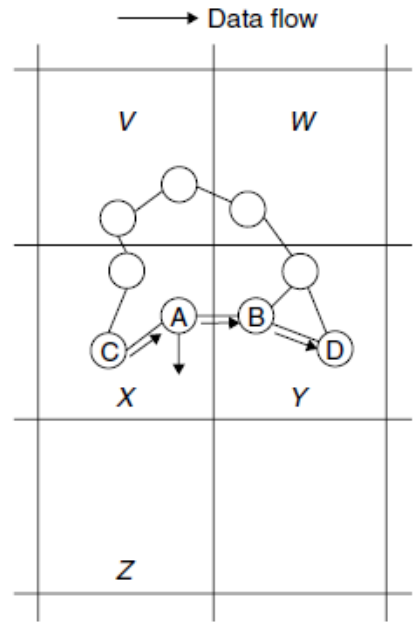

Fig 5.Example of zone-based routing.( Original connectivity between $\mathrm{C}$ and $\mathrm{D}$ ).

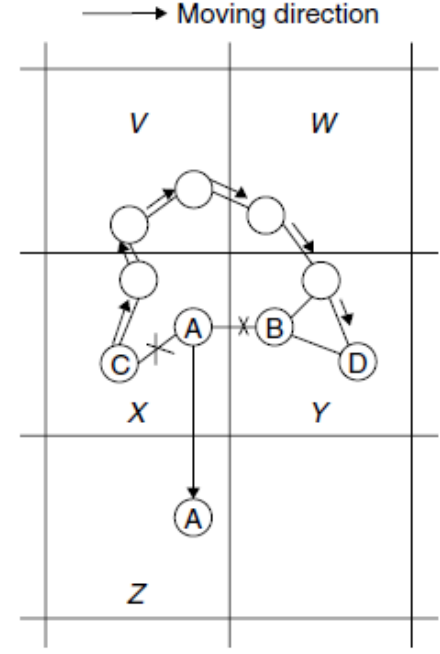

Fig. 6 Connectivity between $C$ and $D$ after $A$ moves to zone $Z$.

Zone Routing partitions the whole network area into squares in advance. Each mobile host knows this partition, so they know their own zone. There are two kinds of routing update in this protocol: intrazone and interzone. Local position change within a square triggers only local link state routing update in the square, while change of connectivity between squares triggers global routing update[13]. For example, host A and host B connect square $X$ and square $Y$, and is the only connection between these two squares (Figure [6]). In this scenario and B are called 
gateways of square $X$ and square $Y$, respectively. Node $\mathrm{C}$ in zone $X$ and node $\mathrm{D}$ in zone $Y$ share the route $\mathrm{C}-\mathrm{A}-\mathrm{B}-$ D.When A moves from zone $X$ to zone $Y$, it loses the connectivity with B, the connectivity between square $X$ and square $Y$ is also lost. This change will trigger the global routing information update. After that, $\mathrm{C}$ must resort to zone $V$ and zone $W$ to communicate with $\mathrm{D}$.

\section{Intra zone routing Protocol}

The construction of a routing zone requires a node to first know who its neighbors are. A neighbor is defined as a node with whom direct (point-to-point) communication can be established and is, thus, one hop away. Identification of a node's neighbors may be provided directly by the media access control (MAC) protocols, as in the case of polling-based protocols. In other cases, neighbor discovery may be implemented through a separate Neighbor Discovery Protocol (NDP). Such a protocol typically operates through the periodic broadcasting of hello" beacons. The reception (or quality of reception) of a "hello" beacon can be used to indicate the status of a connection to the beaconing neighbor. Neighbor discovery information is used as a basis for the IARP. IARP can be derived from globally proactive link state routing protocols that provide a complete view of network connectivity[20].

\section{Inter zone routing Protocol}

Example for interzone routing protocol is Bordercast Resolution Protocol. Route discovery in the Zone Routing framework is distinguished from standard broadcast-based route discovery through a message distribution service known as the Bordercast Resolution Protocol (BRP). Rather than blindly broadcasting a route query from neighbor to neighbor, bordercasting allows the query to be directed outward, toward regions of the network (specifically, toward peripheral nodes)that have not yet been "covered" by the query. (A covered node is one that belongs to the routing zone of a node that has received a route query). The query control mechanisms reduce route query traffic by directing query messages outward from the query source and away from covered routing zones[20].

A node can determine local query coverage by noting the addresses of neighboring nodes that have forwarded the query. In the case of multiple channel networks, a node can only detect query packets that have been directly forwarded to it. For single channel networks, a node may be able to detect any query packet forwarded within the node's radio range. When a node identifies a query forwarding neighbor, all known members of that neighbor's routing zone (i.e.,those members which belong to both the node's and neighbor's routing zones) are marked as covered.
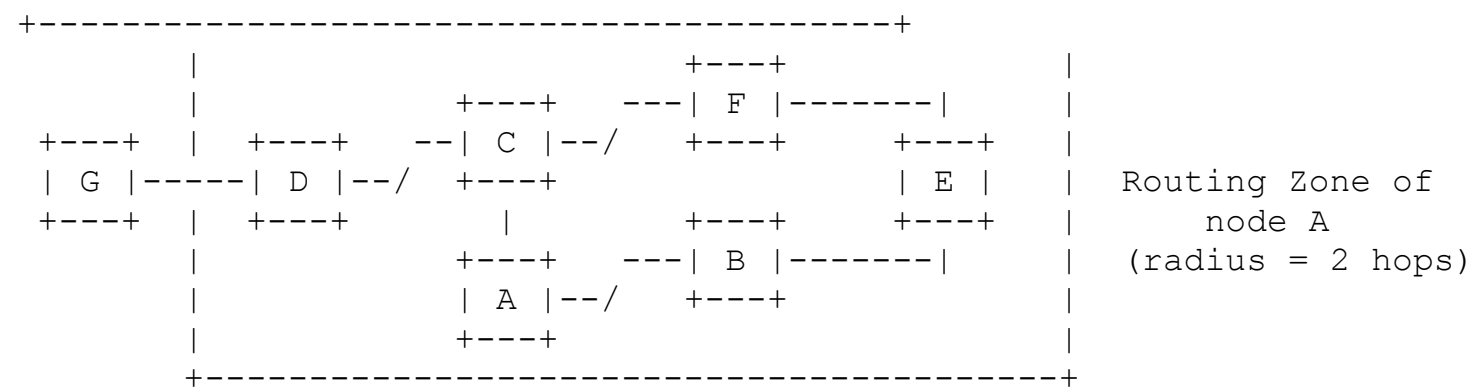

Fig.7 An example for Zone Routing

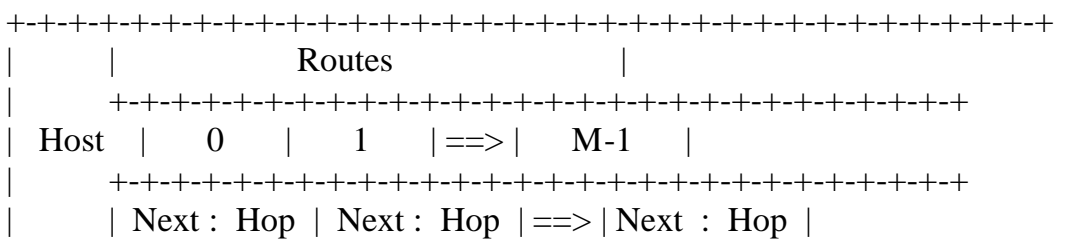




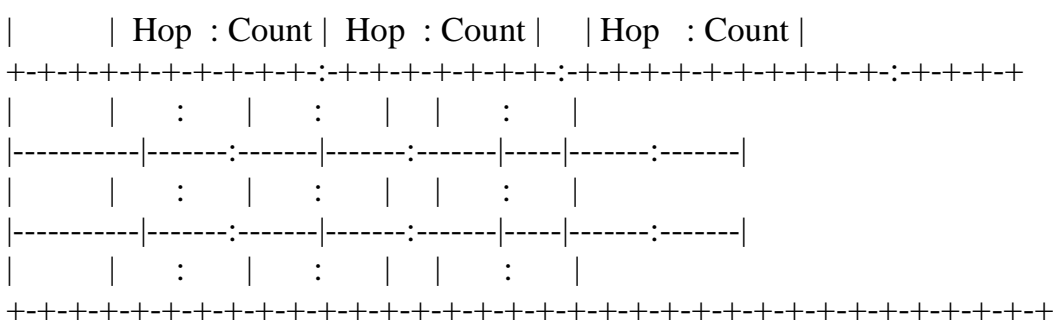

Fig.8 Structure of Intrazone Routing

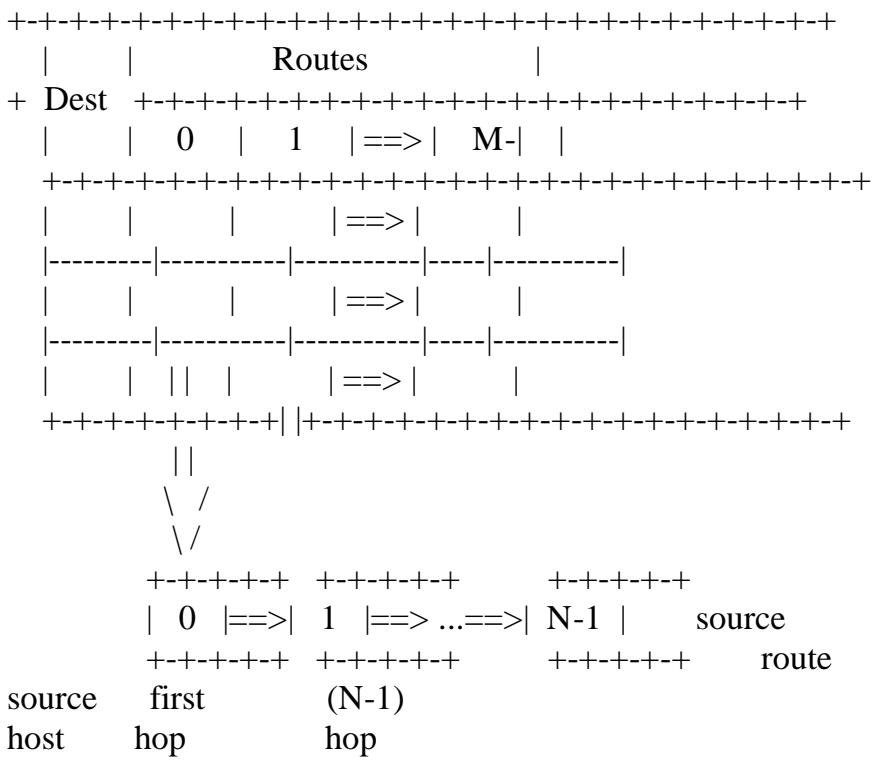

Fig.9 Structure of Interzone Routing Table

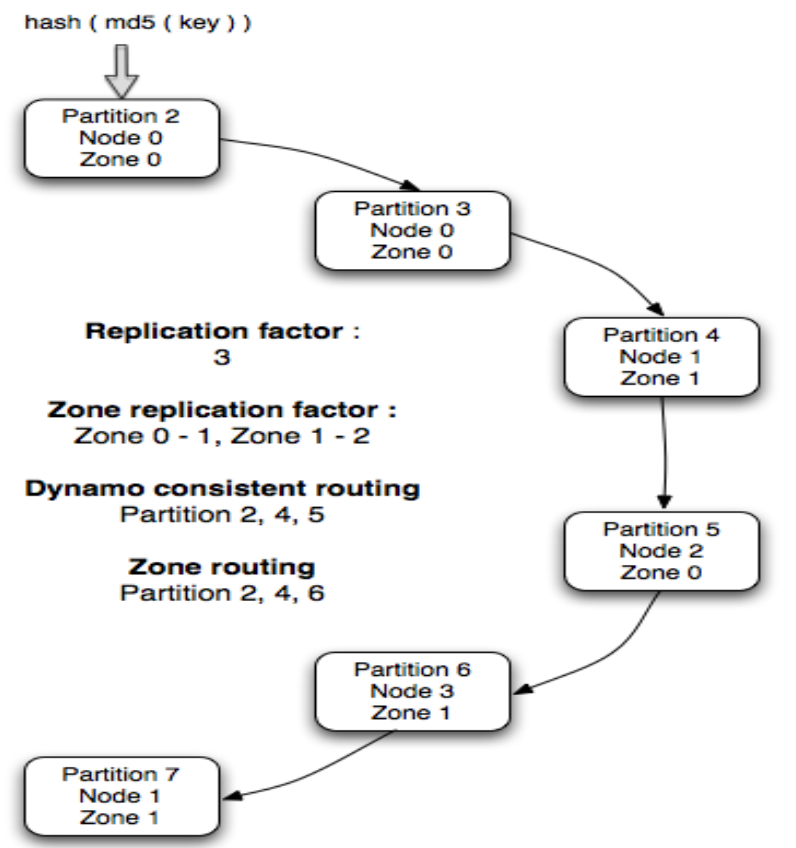

Fig.10 Structure of Zone Routing with Replication Factor

Replication-Factor - This is the total number of times the data is stored. Each put or delete operation must eventually hit this many nodes. A replication factor of $n$ means it can be possible to tolerate up to $n-1$ node failures without data loss.

VI. Algorithm For The Proposed Idea 
1) The source $S$ wants to send data packet to the destination $D$.

2) The destination node is a member of the zone z3 among 5 zones. So, the packet can be sent to any of themember of the zone which is more nearer to the source $S$.

3) The source $S$ checks its IAR packets which are sent periodically to all the nodes within its zone. If therouting information for any of the addressis found using IARpackets then the search isstopped and Step 6 and Step 7 is followed.

4) If the destinationaddress is not found within its zone, then IER packets is border-cast to all theborder nodes of $\mathrm{S}$. If the routing information of the address is found using IER packetsthen the search is stopped and Step 7 and Step 8 is followed.

5) If the destinationaddress is also not found within the previously border-cast nodes, then IER packetsare again border-cast to all the border nodes of that previously border-cast nodes.

6) If address is found, then the IER RouteReply packet is sent from the destination address to the source.

7) The data packets are sent from the source $S$ to the destination $D$ via the information receivedfrom the RouteReply packet.

VII. Simulation Analysis

\begin{tabular}{|l|l|}
\hline \multicolumn{1}{|c|}{ Parameters } & \multicolumn{1}{c|}{ Values } \\
\hline Number of nodes & Ranges from 1000 to 4000 \\
\hline Type of topology & User-specific \\
\hline Network coverage area & $1000[\mathrm{~m}] \mathrm{x} 1000[\mathrm{~m}]$. \\
\hline Transmission radius & $100[\mathrm{~m}]$ \\
\hline Transmission rate & $10.0[\mathrm{Mbps}]$ \\
\hline Number of data packets & Ranges from 100 to 500. \\
\hline $\begin{array}{l}\text { Routing Zone radius (Hop } \\
\text { Count) for ZRP }\end{array}$ & 1 to10 hops \\
\hline Node speed & $10-75[\mathrm{~m} / \mathrm{sec}]$. \\
\hline
\end{tabular}

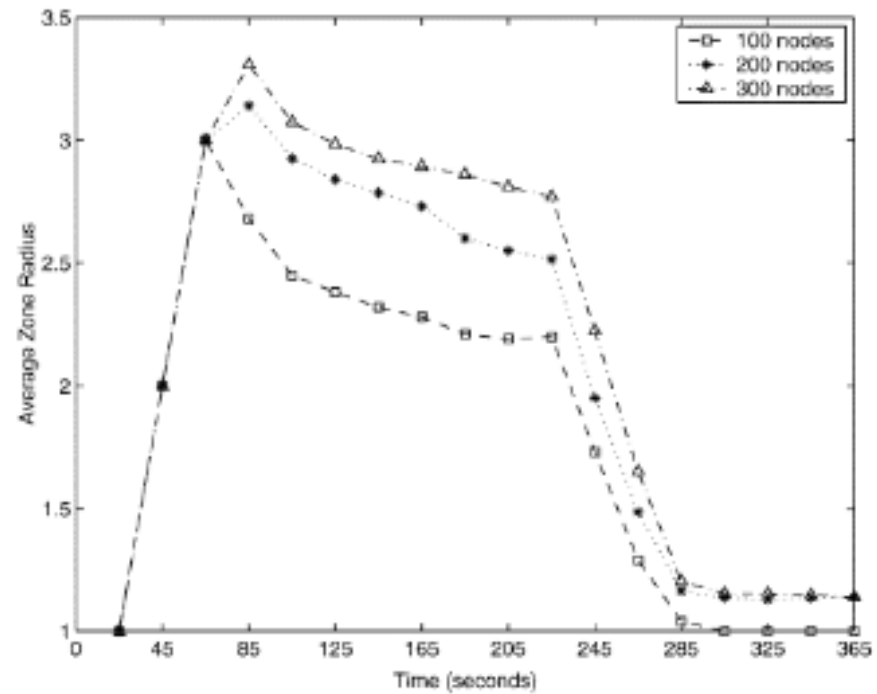

Fig.13 Comparison of throughput of ZRP in different node density by varying Zone radius.

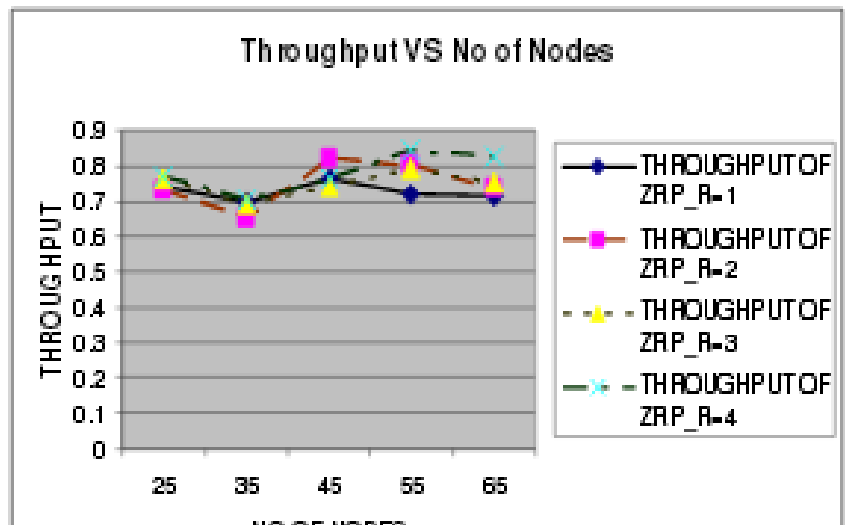


Fig. 11.The average zone radius of the nodes.

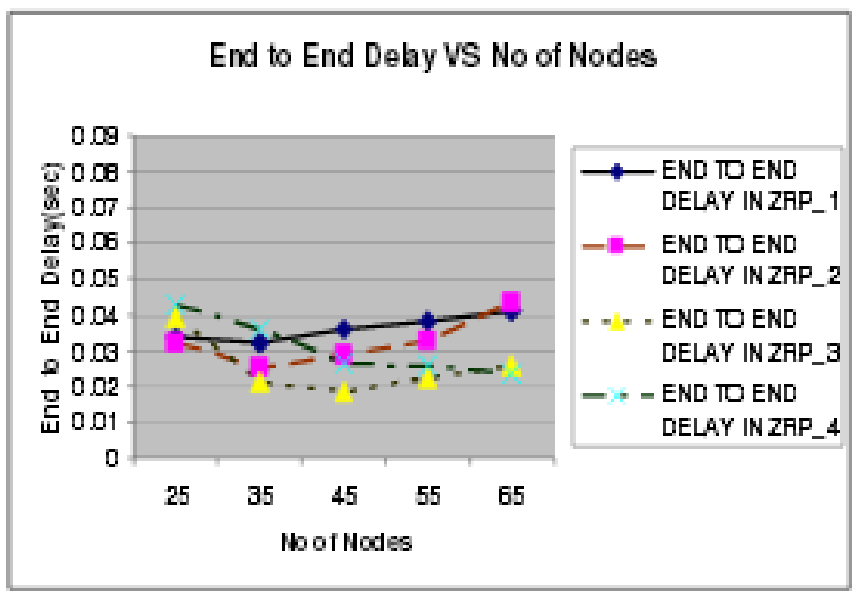

Fig.12 Routing control traffic, as the network size is increased

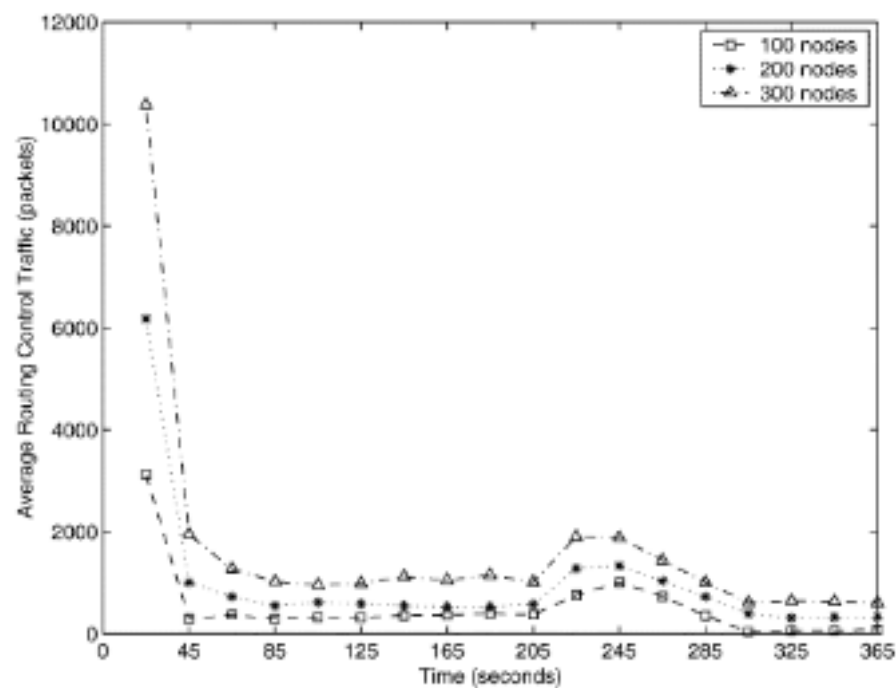

Fig14.Comparison of End to End delay of ZRP in different node density by varying Zone radius. 


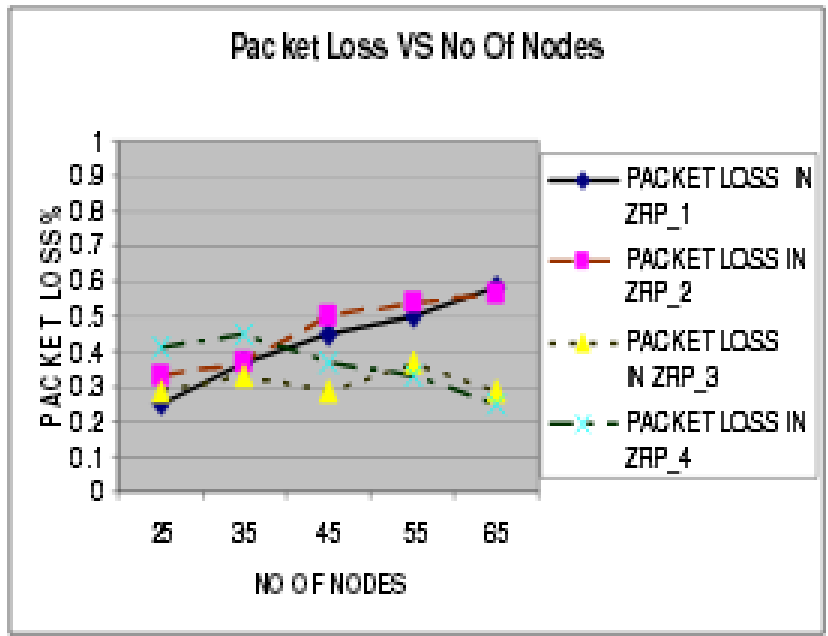

Fig15. Comparison of packet loss using ZRP in different node density by varying Zone Radius

\section{Conclusion}

In this paper a new method was proposed to increase the performance of WEAC Protocol using zone routing algorithm. This zone routing method provides a simple way for creating zones and transmitting video files to the nearby nodes within the zone. This method is tested using less number of packets and less power consumption. The future scope of this WEAC protocol with zone routing algorithm is to successfully simulate it for a very large network and implement it with video application to study the performance and efficiency.

The Result shows that Average zone, control traffic of zone routing, different node density for varying zone, end to end delay for zone routing, packet loss of zone routing. All of the result shows that the performance of zone routing is better than other methods. Hence Zone routing is suitable for video transmission over WEAC Protocol.

\section{References}

[1] Tapaswini Dash et al. Zone Routing Protocol Using Anycast Addressing For Ad-Hoc Network International Journal on Computer Science and Engineering (IJCSE) june 2012

[2] V. D. Park and M. S. Corson, "A highly adaptive distributed routing algorithm for mobile wireless networks, " presented at the IEEE INFOCOM, Kobe, Japan, Apr. 1997.

[3] B. Bellur and R. G. Ogier,"A reliable, efficient topology broadcast protocol for dynamic networks," presented at the IEEE INFOCOM, Mar. 1999.

[4] Z. J. Haas and M. R. Pearlman, "The performance of query control schemes for the zone routing protocol," IEEE/ACM Trans. Networking, vol. 9, pp. 427,438, Aug. 2001.

[5] Z. J. Haas, M. R. Pearlman, and P. Samar, "The bordercast resolution protocol (BRP) for ad hoc networks," IETF, MANET Internet Draft, July 2002. [6]"The interzone routing protocol (IERP) for ad hoc networks," IETF, MANET Internet Draft, July 2002.

[7] A. Iwata, C.-C.Chiang, G. Pei, M. Gerla, and T.-W. Chen, "Scalablerouting strategies for ad hoc wireless networks," IEEE J. Select. Areas Commun., vol. 17, pp. 1369-1379, Aug. 1999.

[8] D. B. Johnson and D. A. Maltz, "Dynamic source routing in ad hoc wireless networking," in Mobile Computing, T. Imielinski and H. Korth, Eds. Boston, MA: Kluwer, 1996.

[9] B. Liang and Z. J. Haas, "Hybrid routing in ad hoc networks with a dynamic virtual backbone," IEEE Trans. Wireless Commun., to be pub-lished.

[10] A. B. McDonald and T. Znati, "Predicting node proximity in Ad-Hoc networks: A least overhead adaptive model for electing stable routes,"presented at the MobiHoc 2000, Boston, MA, Aug. 2000.

[11] J. Moy,"OSPF version 2," IETF, RFC 2178, Mar. 1997.

[12] S. Murthy and J. J. Garcia-Luna-Aceves, “An efficient routing protocol for wireless networks, ”MANET, vol. 1, no. 2, pp. 183-197, Oct. 1996.

[13] M. R. Pearlman, Z. J. Haas, and S. I. Mir, "Using routing zones to support route maintenance in ad hoc networks," presented at the IEEE WCNC 2000, Chicago, IL, Sept. 2000.

[14] M. R. Pearlman, J. Deng, B. Liang, and Z. J. Haas, “ Elective participation in ad hoc networks based on energy consumption," presented at the IEEE GLOBECOM, Nov. 2002.

[15] M. R. Pearlman and Z. J. Haas, "Determining the optimal configuration for the zone routing protocol," IEEE J. Select. Areas Commun., vol. 17, pp. 1395 - 1414, Aug. 1999.

[16] M. Gerla, X. Hong, and G. Pei, "Landmark routing for large ad hoc wireless networks," presented at the IEEE GLOBECOM, San Francisco,CA, Nov. 2000.

[17] G. Pei, M. Gerla, and T.-W. Chen,"Fisheye state routing: A routing scheme for ad hoc wireless networks," presented at the IEEE ICC 2000, New Orleans, LA, June 2000.

[18] G. Pei, M. Gerla, X. Hong, and C.-C. Chiang, "A wireless hierarchical routing protocol with group mobility," presented at the IEEE WCNC '99, New Orleans, LA, Sept. 1999.

[19] C. E. Perkins and P. Bhagwat, "Highly dynamic destination-sequenced distance-vector routing (DSDV) for mobile computers," Proc. ACM SIGCOMM , vol. 24, no. 4, pp. 234-244, Oct. 1994.

[20] T. Clausen and P. Jacquet, “Optimized link state routing protocol(OLSR), ’IETF, RFC 3626, Oct. 2003. 